\title{
\|MPRSSION MATER\|ALS
}

\section{Panasil Binetics}

Panadent is pleased to launch Kettenbach's new Panasil Binetics addition cure impression material. Panadent claims it is the first true putty to be launched in a foil bag suitable for automatic dispensing.

Automatic mixing of putty impression material obviates traditional hand kneading which means potential contamination is avoided, hygiene levels lifted and more consistent results are obtained with less wastage of material.

Panasil claims the Binetics putty Fast Set cures in just 2 mins 30 secs intraorally and is kiwi green in colour so providing ideal contrast to Panasil Contact Plus. For more information phone 01689881788. Reader response number 56

\section{A1 Cool Temp}

A new Cool Temp shade is now available from Coltene Whaledent. The Cool Temp shade A1 has a significantly lower curing temperature. Coltene Whaledent claims it does not damage or irritate the pulp or gingiva, and the fast set gives a material which is quick to trim and polish.

Coltene Whaledent also claims that the Cool Temp gives precise "temporaries" without extensive chairside adjustments. Priced at $£ 94.95$ the introductory pack is available from your dealer now, or phone 0500295454 for further details.

Reader response number 57

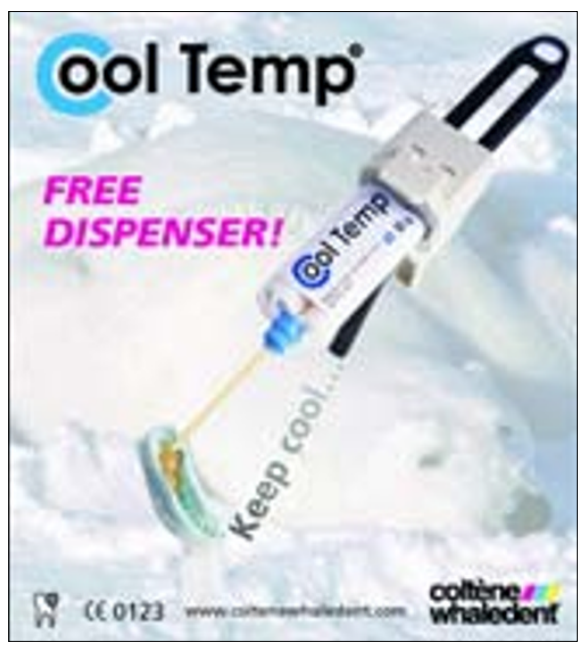

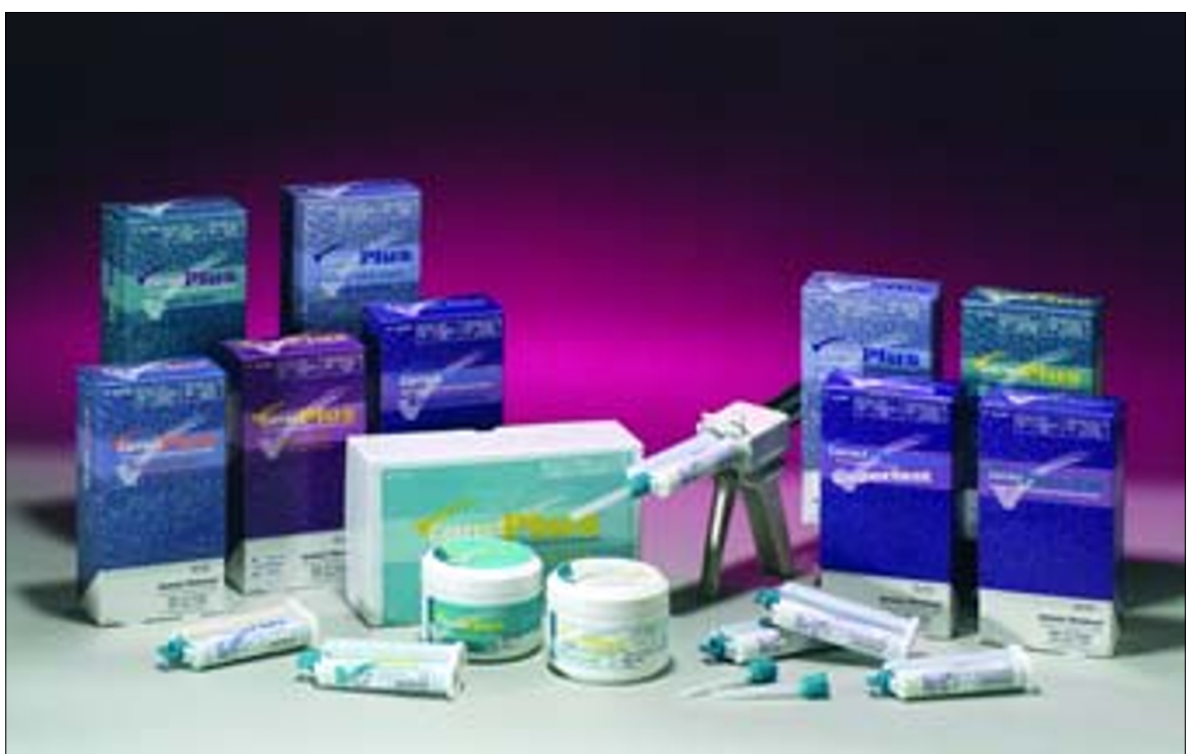

\section{Get your Thick nThin putty from Trycare}

Part of Trycare's Correct Plus range is the Thick nThin putty. Trycare describes it as a light body material that syringes onto the teeth like a medium body, but is thinned by the heat of the teeth so that it automatically flows into the sulcus to capture critical sub-gingival details.

Trycare also says that it possesses excellent tear strength and detail reproduction. Trycare claim that it is ideal for the putty/wash, dual arch and heavy/light techniques.

The Correct Plus Range of hydrophilic impression materials also incorporate a syringe material, monophase material, Injectable putty and bite tray material all offering innovative viscosities to enhance any technique.

For further details of the Correct Plus Range or other Jeneric Pentron products contact Trycare on 01274881044.

Reader response number 55

\section{The Centric Tray from Ivoclar Vivadent}

The new Centric Tray from Ivoclar Vivadent provides a quick and simple solution for taking a preliminary record of the patient's individual centric and vertical relationship, and is an ideal complement to the Biofunctional Prosthetics System.

The resulting impression is used as a matrix to transfer information about centric relation to the dental laboratory. Existing models can then be accurately mounted on the articulator, and subsequent steps to attaining an accurate centric relation record are made significantly easier.

Ivoclar also claims that as well as reducing the need for subsequent adjustments, the Centric Tray also helps save time and money, as it can be autoclaved and used several times.

Further information phone Ivoclar Vivadent on 01162654055.

Reader response number 58

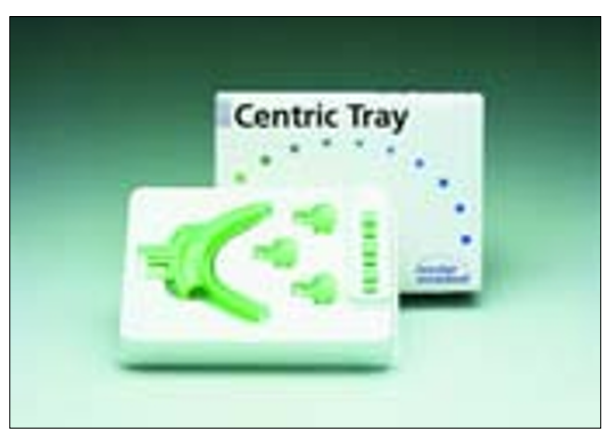




\section{Get a creamy consistency from Dentsply}

The Blueprint Cremix from Dentsply is a lead-free, easy mixing alginate. The mix is suitable for full and partial oral impressions, including those for orthodontics, prosthetics, occlusion analyses and study models.

Dentsply claims the light, wettable, Blueprint Cremix powder results in a creamy consistency. The dust-free nature ensures no eye irritation or powder inhalation when mixing. They also claim that, coupled with a lead-free product, means it is safe in every way.

Also available are the alginate mixing bowls, Ash alginate spatulas and Ash Poly Trays - disposable impression trays available in assorted sizes.

For more information phone 01932853422.

Reader response number 59

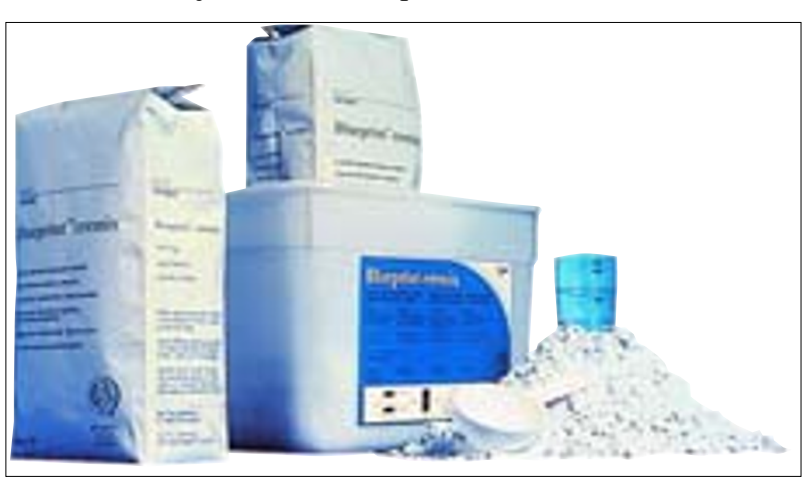

\section{The Wafer thin Impression material from GC}

GC claims that its Impression Wafers will revolutionise the way dentists take impressions by allowing them to take far more accurate crown and bridge impressions than ever before.

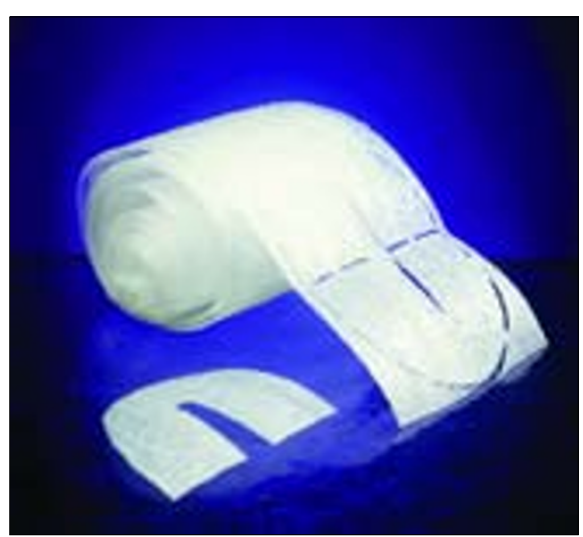

The hydrophilic qualities of the material enhance the wettability by reducing contact angles and surface tension. This enables a uniform flow of the impression material over moist surfaces to produce an exact replication.

GC also claims that the Exafast sets quickly in just in 2 minutes 15 seconds and the impressions are easily removed from undercut areas without the risk of tearing or permanent deformation. This technique will save any re-takes, and allows for perfect fitting crowns, bridges, inlays and veneers with little adjustment to the final restoration.

For further information please contact GC UK on 01908 218999, e-mail gcuk@btinernet.com or visit www.gceurope.com.

Reader response number 60

\section{Neocolloid and Hydrogum from Zhermack}

Zhermack have two non-silicone solutions. Firstly Neocolloid, a unique high detail alginate suitable for chrome work. Secondly, Hydrogum, which gives good reproduction, and with a faster setting time is a more appropriate alginate for all prosthetic and orthodontic indications.

Zhermack claims both alginates are easy to mix, and when set, dentists could easily be mistaken for thinking they were rubber based materials. They have a smooth surface, good elasticity, and the high alginate gel content, gives the material extra strength and tear resistance.

Also available is Trialgin, a colour changing version of Hydrogum with a mango flavour. Trialgin is red to mix, goes salmon pink when it is time to put it in the tray and yellow when it is time to insert in the mouth.

For more information phone 07870690811.

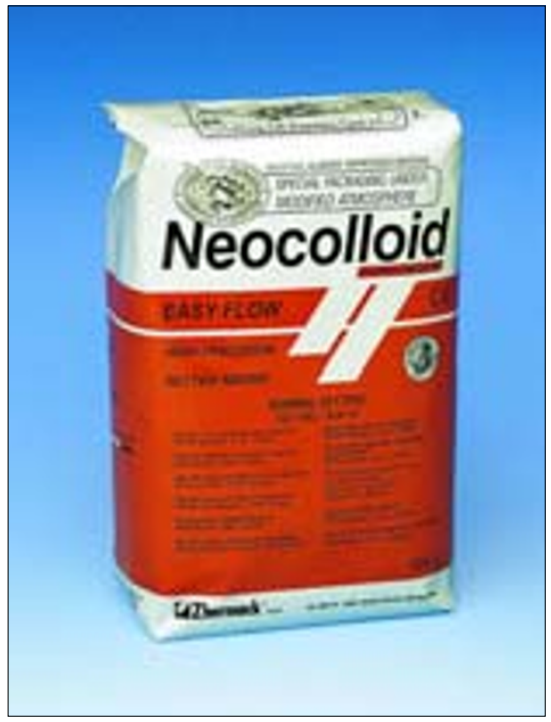

Reader response number 61 